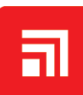 \\ CHITKARA \\ Journal of Nuclear Physics, Material Sciences, Radiation and Applications \\ Journal homepage: https://jnp.chitkara.edu.in/
}

\section{Comparative Analysis of Woods-Saxon and Yukawa Model Nuclear Potentials}

\author{
O.S.K.S. Sastri iD, Aditi Sharma iD, Swapna Gora iD and Richa Sharma \\ Central University of Himachal Pradesh, Dharamshala, Himachal Pradesh - 176206, India \\ "sastri.osks@hpcu.ac.in (Corresponding Author)
}

\section{ARTICLE INFORMATION}

Received: January 8, 2021

Accepted: March 30, 2021

Published Online: August 31, 2021

\section{Keywords:}

Nuclear potential, Rephrasing of potentials,

Magic nuclei

DOI: $10.15415 /$ jnp.2021.91013

\section{ABSTRACT}

In this paper, we model the nuclear potential using Woods-Saxon and Yukawa interaction as the mean field in which each nucleon experiences a central force due to rest of the nucleons. The single particle energy states are obtained by solving the time independent Schrodinger wave equation using matrix diagonalization method with infinite spherical well wave-functions as the basis. The best fit model parameters are obtained by using variational Monte-Carlo algorithm wherein the relative mean-squared error, christened as chi-squared value, is minimized. The universal parameters obtained using WoodsSaxon potential are found to be matched with literature reported data resulting a chi-square value of 0.066 for neutron states and 0.069 for proton states whereas the chi-square value comes out to be 1.98 and 1.57 for neutron and proton states respectively by considering Yukawa potential. To further assess the performance of both the interaction potentials, the model parameters have been optimized for three different groups, light nuclei up to ${ }^{16} \mathrm{O}-{ }^{56} \mathrm{Ni}$, heavy nuclei ${ }^{100} \mathrm{Sn}-{ }^{208} \mathrm{~Pb}$ and all nuclei ${ }^{16} \mathrm{O}-{ }^{208} \mathrm{~Pb}$. It is observed that Yukawa model performed reasonably well for light nuclei but did not give satisfactory results for the other two groups while Woods-Saxon potential gives satisfactory results for all magic nuclei across the periodic table.

\section{Introduction}

One of the successful models for explaining the sudden increase in binding energy, at $\mathrm{N}$ or $\mathrm{Z}=2,8,20,50,82$ and 126 called as magic numbers, is the nuclear shell model [1]. The interaction is modeled as harmonic oscillator, a central mean field potential experienced by each nucleon due to rest of the nucleons. The actual shell closures were obtained only after including the spin-orbit interaction that is introduced as proportional to derivative of the mean field potential, whose experimental evidence has been found later [2]. There are other potential which have been suggested such as square well, cosh geometry [3] given by

$$
V_{N}(r)=-V_{0} \frac{1+\cosh \left(\frac{R}{a}\right)}{\cosh \left(\frac{r}{a}\right)+\cosh \left(\frac{R}{a}\right)}
$$

where $V_{0}$ is the depth of the well, $R$ is radius of the nucleus and $a$ is the diffuse parameter, but the most successful Woods-Saxon potential [4] for explaining shell closures is given as

$$
V_{W S}(r)=\frac{V_{0}}{1+\exp \left(\frac{r-R}{a}\right)}
$$

where

$$
V_{0}=\left\{\begin{array}{l}
-V+k((N-Z) / A) M e V, \text { for Neutrons } \\
-V-k((N-Z) / A) M e V, \text { for Protons }
\end{array}\right.
$$

$R=1.28 A^{(1 / 3)} \mathrm{fm}, A$ is the mass number of the nucleus and $a=0.66 \mathrm{fm}$.

Fundamentally, the interaction between nucleons is best understood by the Yukawa potential. Gauthier [4] has utilized this fundamental interaction to explain the emergence of volume and surface terms in semi-empirical mass formula, which are otherwise classically modeled on the analogy of liquid drop model. Later, this methodology has been extended to provide basis for mean-field nuclear potential in the Yukawa model [5] where, net potential for neutrons is given by

$$
V_{N}(r)=\left\{\begin{array}{c}
-V_{0}\left[1-\left(1+\frac{R}{\lambda}\right) e^{\frac{-R}{\lambda}} \frac{\sinh \left(\frac{r}{\lambda}\right)}{\left(\frac{r}{\lambda}\right)}\right], \text { when } r \leq R \\
V_{0} \lambda\left[\left(\frac{R}{\lambda}\right) \cosh \left(\frac{R}{\lambda}\right)-\sinh \left(\frac{R}{\lambda}\right)\right] \frac{e^{\frac{-r}{\lambda}}}{r}, \text { when } r \geq R
\end{array}\right.
$$


with $V_{0}=3 g^{2} \frac{\lambda^{2}}{R_{0}^{3}}$

Here, $g^{2}$ is the universal constant, of order of $0.1 \hbar c$ ( 20 $\mathrm{MeV}-\mathrm{fm}$ ), which represents strength of nuclear force, and $\lambda$ represents interaction range, it has a value of the order of $1.43 \mathrm{fm}$. But for reproducing all magic nuclei numbers, spin orbit potential $V_{k}$ has to be added to mean field potential whose functional form is given by

$$
V_{l s}(r)=\left(\frac{r_{0}}{\hbar}\right)\left[\frac{d}{d r}(V(r))\right](L \cdot S)
$$

In addition to this, for protons states, Coulomb interaction has to be included which is introduced as:

$$
V_{C}(r)=Z^{\prime} e /\left(4 \pi \varepsilon_{0} r\right) ; \text { for } \geq R_{c}
$$

Inside the nucleus $(r<R)$, the potential is given by:

$$
V_{C}(r)=\frac{1}{2}\left(\frac{Z^{\prime} e}{4 \pi \varepsilon_{0} r}\right)\left(3-\frac{r^{2}}{R_{c}^{2}}\right) ; \text { for } r \leq R_{C}
$$

where $Z^{\prime}=Z-1, e$ is the charge of proton and $\varepsilon_{0}$ is the permittivity of vacuum.

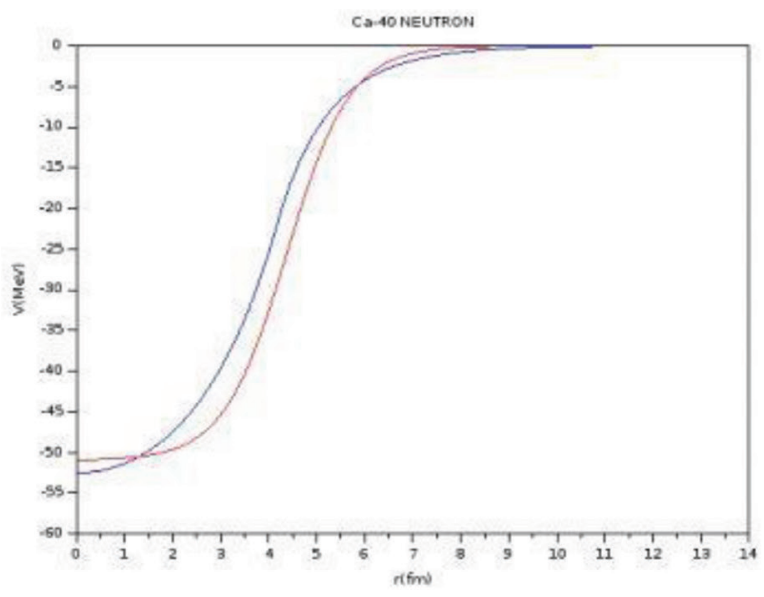

While plotting and neutrons and proton states for ${ }^{40} \mathrm{Ca}$ nuclei, both Yukawa and Woods-Saxon potentials shows good resemblance with each other and is shown in Figures 1(a) and (b) respectively. Our PER group has already solved the time independent Schrodinger equation (TISE) using the matrix method as proposed by Marsiglio et al., [6,7] for various potentials such as square well [8], anharmonic [9], Morse [10] and Woods-Saxon [11]. Recently, we have optimized the model parameters for the Morse potential by proposing a variational Monte-Carlo (VMC) technique [12]. We have also utilized this Morse potential to model the $\mathrm{n}-\mathrm{p}$ interaction in deuteron and obtained the scattering phase shifts all the way up to 300 $\mathrm{MeV}$ successfully [13].

In this paper, our main objectives are:

1. To determine the single particle neutron and proton energies using both Woods-Saxon and Yukawa models utilizing matrix methods [6] and

2. To optimize the model parameters using variational Monte-Carlo technique [12] by minimizing the relative mean-square error, christened as chi-squared error.

In Section 2, the simulation methodology is briefly described and the results are discussed in detail in Section 3. Finally, we draw our conclusions in Section 4.

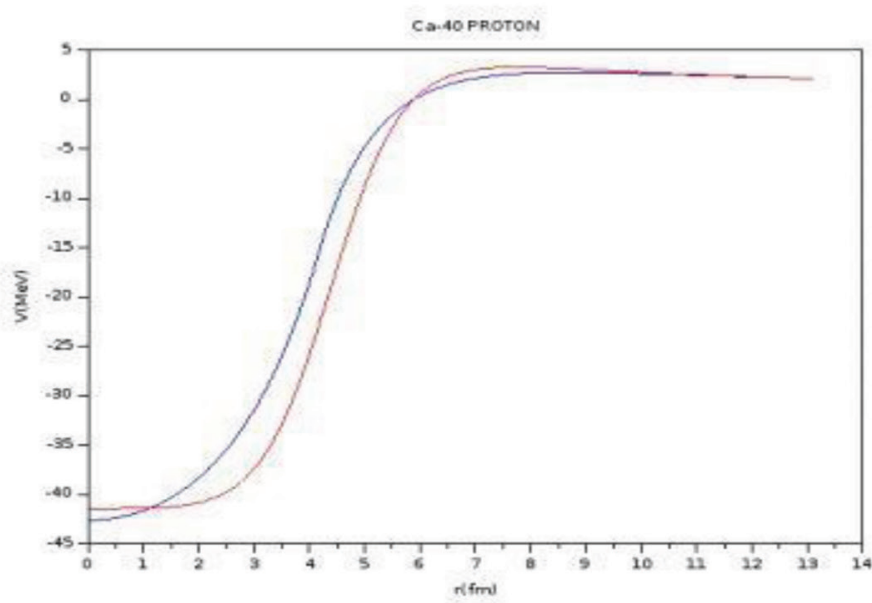

Figure $1: \mathrm{V}(\mathrm{MeV})$ vs $\mathrm{r}(\mathrm{fm})$ plots of Ca-40 for (a) Proton and (b) Neutron. Red and blue curve represent Woods-Saxon and Yukawa potentials respectively.

\section{Simulation Methodology}

\subsection{Preparation of System for Numerical} Solution

Choice of numerical technique: Here, we utilize Marsiglio matrix diagonalization technique whose central idea is to embed any potential of interest within an infinite square well potential of width ' $a$ ' i.e using simple sine basis. The time independent Schrodinger equation (TISE) in Dirac notation is written as

$$
H|u \geq E| u>
$$

where

$$
H=H_{0}+V(r)=\frac{-h^{2}}{2 \mu} \frac{d^{2}}{d r^{2}}+V_{\text {inf }}(r)+V_{\text {eff }}(r)
$$


Here, $V_{\text {inf }}(r)$ corresponds to infinite square well potential and the effective potential $\mathrm{V}_{\text {eff }}(\mathrm{r})$ is defined as $V_{\text {eff }}(r)=V(r)+V_{\text {c.f. }}(r)+V_{l s}(r)$

where

$$
V_{c . f .}(r)=\frac{l(l+1)}{2 \mu r^{2}}
$$

Choosing eigen functions of $\mathrm{H}_{0}, \varphi_{n}(r)=\sqrt{\frac{2}{a_{0}}} \sin \left(\frac{n \pi r}{a_{0}}\right)$ as basis functions, the wave function $\mathrm{u}(\mathrm{r})$ is written as linear combination of $\varphi_{n}(r)$ as

$$
\left|u>=\sum_{n=1}^{\infty} c_{n}\right| n>
$$

where $c_{n}$ are the Fourier coefficients. Now substituting Eq. (4) in Eq. (1) following inner product with $<\mathrm{m} \mid$ and using orthonormality condition, final eigen value equation in matrix form is obtained as

$$
\sum_{n=1}^{\infty} H_{n m} c_{n}=E c_{m}, m=1,2,3, \ldots
$$

Here, $H_{n m}$ is the matrix of infinite dimensions. To make it finite size, we prefer to choose $N_{0}$ as number of basis functions.

\subsection{Rephrasing of Potentials in Appropriate Units}

The Woods-Saxon, Yukawa and spin-orbit potentials are already defined in $\mathrm{MeV}$. So, we need to rephrase remaining two potentials only.

(i) Rephrasing of centrifugal potential: $V_{c f}$ is the centrifugal term, which increases as the orbital angular momentum of nucleon increases and is rephrased by multiplying both numerator and denominator with a factor of $\mathrm{c}^{2}$ and taking $\hbar \mathrm{c}=197.329 \mathrm{MeV}$-fm as

$$
V_{c . f .}=\frac{l(l+1)(197.329)^{2}}{2 \mu c^{2} r^{2}}
$$

The reduced mass, $\mu$ is

$$
\mu=\left\{\begin{array}{l}
\frac{m_{n}\left(Z m_{p}+(N-1) m_{n}\right)}{\left(Z m_{p}+N m_{n}\right)} ; \text { for neutrons } \\
\frac{m_{p}(Z-1) m_{p}+(N) m_{n}}{\left(Z m_{p}+N m_{n}\right)} ; \text { for protons }
\end{array}\right.
$$

where $m_{p}$ is mass of proton $\left(=938.272 \mathrm{MeV} / \mathrm{c}^{2}\right)$ and $m_{n}$ is mass of neutron $\left(=939.565 \mathrm{MeV} / \mathrm{c}^{2}\right)$

(ii) Rephrasing of Coulomb potential: The coulomb potential defined in Section 1 is redefined as

$$
V_{C}(r)=\left\{\begin{array}{c}
\frac{Z-1 \times 0.511 \times 2.839}{r}, \text { if } r \geq R_{C} \\
\frac{(Z-1) \times 0.511 \times 2.839}{R_{C}}\left(\frac{3}{2}-\frac{r^{2}}{2 R_{C}}\right), \text { if } r \leq R_{C}
\end{array}\right.
$$

The system has been solved using matrix methods technique utilizing the code written[11] for obtaining the single particle energies by solving TISE for Woods-Saxon mean field potential and by redefining the potentials for $V_{N}(r)$ and $V_{L S}(r)$ as per the Yukawa model. The optimization of model parameters has been done by using the variational MonteCarlo technique code.

\section{Results and Discussion}

\subsection{Optimization of Woods - Saxon Parameters using Monte-Carlo Approach}

The Monte-Carlo algorithm is based on varying the model parameters randomly by small amount while variational approach involves minimizing the chi-square value in each iteration. In order to start with, WS parameter values have to be initialized firstly for neutron states which includes $V_{0}$, a, $\mathrm{R}_{0}$, kappa. Since all these parameters are optimized earlier for ${ }^{40} \mathrm{Ca}$ and ${ }^{208} \mathrm{~Pb}$ nuclei together in [11], therefore these are taken as initial guess for further optimization considering all doubly magic nuclei across the periodic table. Using these values, TISE is solved using matrix diagonalization technique as discussed earlier and energy values are determined. Now, we define relative mean square error, and call it the chi-square value which is to be minimized for obtaining convergence with the experimental data, as

$$
X^{2}=\frac{1}{N} \frac{\sum_{i=1}^{N}\left(E_{\text {exp. }}^{i}-E_{\text {num. }}^{i}\right)^{2}}{\left|E_{\exp _{i}}\right|}
$$

where $\mathrm{N}$ is the number of experimental energy levels available for a chosen nucleus. Here experimental data is taken from [14]. In each iteration, one of the parameters has been chosen randomly and has been varied by a value that has been randomly generated within an interval [-I, I] . This interval range is decremented after a certain number of iterations when the change in chi-square value is not substantial. The process is repeated till the chi-square value is far less than 1 . The final optimized neutron parameters are given as input for calculation of proton states along with the addition of one more parameter i.e. charge radius, Rc which is set as 1.2 close to literature reported value. Again, VMC calculations were repeated for proton states taking all nuclei together. The optimized parameters along with chi-square value of neutron and proton levels taking all magic nuclei together are given in Table 1. 
Table 1: Chi-square values for groups of doubly magic nuclei across the periodic table for Woods-Saxon potential.

\begin{tabular}{llllllll}
\hline Neutron/Proton & $\mathbf{V}_{0}$ & Kappa & $\mathbf{a}$ & $\mathbf{R}_{0}$ & $\mathbf{r}_{0}$ & $\mathbf{R}_{\mathrm{c}}$ & chi-square \\
\hline Neutron & 51.48628 & 0.781218 & 0.5900557 & 1.286356 & 0.9194008 & - & 0.0661832 \\
Proton & 53.02575 & 0.5621975 & 0.598853 & 1.27966 & 0.802733 & 1.1210415 & 0.069
\end{tabular}

Similar calculations were repeated for Yukawa potential also. A total of five model parameters $\lambda, \mathrm{g}^{2}, \mathrm{r}_{0}, \mathrm{R}_{0}$ and $\mathrm{R}_{\mathrm{C}}$ (charge radius-initially chosen to be $\mathrm{R}_{0}$ ) are present in the simulation which needs to be optimized further.

\subsection{Optimization of Yukawa Parameters using Monte-Carlo Approach}

\subsubsection{For ${ }^{48} \mathrm{Ca}$ and ${ }^{132} \mathrm{Sn}$ Nuclei}

As a first step, we have determined the neutron and proton energy levels for ${ }^{48} \mathrm{Ca}$ nucleus only due to the Yukawa model mean-field potential by choosing model parameters reported in literature as $\lambda=1.43 \mathrm{fm}, \mathrm{g}^{2}=0.1 \mathrm{MeV} \mathrm{fm}, \mathrm{R}_{0}=1.28$ $\mathrm{fm}[2]$ as initial values for optimization. The final optimized parameters obtained after minimizing chi-square value for neutrons are: $\lambda=1.37, \mathrm{~g}^{2}=0.11, \mathrm{R}_{0}=1.295$, and $\mathrm{r}_{0}=0.158$ whereas for protons: $\lambda=1.54, \mathrm{~g}^{2}=0.12$, $\mathrm{R}_{0}=1.295, \mathrm{R}_{\mathrm{c}}=1.27$, and $\mathrm{r}_{0}=0.025$ respectively. Using these values, the single particle neutron and proton energy states are determined using matrix method and are compared with experimental data. The data is tabulated in Table 2 and \%error for each of the state calculated is shown along with chi-square value.

It is observed that, for the single particle neutron energy states of ${ }^{48} \mathrm{Ca}$, energies obtained for various 1 -values from Yukawa potential shows less than $17 \%$ error with chi-square value 0.20 and for proton states the \%error obtained is less than $14 \%$ with chi-square value 0.18 .

Table 2: Determination of Neutron and Proton energy levels of ${ }^{48} \mathrm{Ca}$ nucleus and comparison with experimental available binding energies.

\begin{tabular}{lllll}
\hline & $\begin{array}{l}\text { Neutron/Proton } \\
\text { states }\end{array}$ & Simulated value & $\begin{array}{l}\text { Experimental } \\
\text { value[14] }\end{array}$ & \%error \\
\hline \multirow{3}{*}{ Neutron } & $1 \mathrm{~d} 5 / 2$ & -17.27 & -15.61 & 10.63 \\
& $2 \mathrm{~s} 1 / 2$ & -14.25 & -12.55 & 13.55 \\
& $1 \mathrm{~d} 3 / 2$ & -11.16 & -12.53 & 10.93 \\
& $1 \mathrm{f} 7 / 2$ & -8.33 & -10.00 & 16.7 \\
\hline \multirow{3}{*}{ Proton } & $1 \mathrm{~d} 5 / 2$ & chi-square error & -21.47 & $\mathbf{0 . 2 0}$ \\
& $1 \mathrm{~d} 3 / 2$ & -18.55 & -16.18 & 13.60 \\
& $2 \mathrm{~s} 1 / 2$ & -17.27 & -16.10 & 7.74 \\
\hline & -17.26 & chi-square error & $\mathbf{0 . 1 8}$ \\
\hline
\end{tabular}

\subsubsection{For ${ }^{132} \mathrm{Sn}$ Nucleus}

Next, another doubly magic nucleus ${ }^{132} \mathrm{Sn}$ is considered and the best fitted parameters using VMC technique by minimizing chi-square value for proton were found which are: $\lambda=1.44, \mathrm{~g}^{2}=0.12, \mathrm{R}_{0}=1.29, \mathrm{R}_{\mathrm{c}}=1.25$, and $r_{0}=0.11$ and for neutron are: $\lambda=1.43, \mathrm{~g}^{2}=0.109$, $\mathrm{R}_{0}=1.32$, and $\mathrm{r}_{0}=0.0944$ respectively. Using these values, again single particle energy states for both neutron and protons are computed and are compared with those of corresponding experimental values available in Table 3. It is observed that simulated proton energies match well with experimental values almost perfectly and they remain within $20 \%$ error for neutrons. This process of obtaining optimized parameters has been undertaken for each of the available doubly magic nuclei in the periodic table. It has been observed that the obtained values for single particle energies in all cases are having less than $20 \%$ error mostly when the parameters are optimized for each individual nucleus. Then, we have extended the simulation to obtain universal model parameters by considering three sets of nuclei to study the effectiveness of the Yukawa model in comparison to WoodsSaxon model. 
Table 3: Comparison of neutron and proton single particle energies for ${ }^{132} \mathrm{Sn}$ nucleus with experimental values.

\begin{tabular}{lllll}
\hline & $\begin{array}{l}\text { Neutron/Proton } \\
\text { states }\end{array}$ & Simulated value & $\begin{array}{l}\text { Experimental } \\
\text { value[14] }\end{array}$ & \%error \\
\hline \multirow{4}{*}{ Neutron } & $1 \mathrm{~g} 7 / 2$ & -7.85 & -9.75 & 19.48 \\
& $2 \mathrm{~d} 5 / 2$ & -10.18 & -8.97 & 13.49 \\
& $1 \mathrm{~s} 1 / 2$ & -8.91 & -7.64 & 16.62 \\
& $2 \mathrm{~d} 11 / 2$ & -6.24 & -7.54 & 17.24 \\
\hline \multirow{3}{*}{ Proton } & $2 \mathrm{p} 1 / 2$ & -8.09 & -7.31 & 10.67 \\
\hline
\end{tabular}

\subsection{Optimization for Light, Heavy and all Nuclei}

All available magic nuclei have been grouped into three categories i.e.

(I) Light nuclei from ${ }^{16} \mathrm{O}-{ }^{56} \mathrm{Ni}$

(II) Heavy nuclei from ${ }^{100} \mathrm{Sn}-{ }^{208} \mathrm{~Pb}$

(III) All nuclei ${ }^{16} \mathrm{O}-{ }^{208} \mathrm{~Pb}$ and model parameters are determined for all three groups utilizing variational Monte-Carlo technique, by minimizing the chi-square value in least square sense. The best fit parameters along with chi-square values for each group are shown in Table 4 for neutrons and protons.

Table 4 : Chi- square values corresponding to best fit parameters for groups of doubly magic nuclei across the periodic table.

\begin{tabular}{llllllll}
\hline Neutron/ Proton & Nucleus & $\lambda$ & $\mathbf{g}^{2}$ & $\mathbf{R}_{\mathbf{0}}$ & $\mathbf{R}_{\mathbf{c}}$ & $\mathbf{r}_{\mathbf{0}}$ & chi-square \\
\hline \multirow{3}{*}{ Neutron } & Light & 1.65 & 0.12 & 1.587 & - & 0.05 & 1.11 \\
& Heavy & 1.48 & 0.104 & 1.47 & - & 0.108 & 1.30 \\
& All & 1.41 & 0.11 & 1.38 & - & 0.07 & 1.98 \\
\hline \multirow{2}{*}{ Proton } & Light & 1.45 & 0.135 & 1.28 & 0.60 & 0.07 & 0.42 \\
& Heavy & 1.59 & 0.10 & 1.247 & 0.69 & 0.05 & 0.81 \\
& All & 1.44 & 0.11 & 1.264 & 1.22 & 0.07 & 1.57 \\
\hline
\end{tabular}

A few observations are in place here. Firstly, the value of $\lambda$ is way away from experimental one of 1.43 in case of light nuclei for neutrons (1.65) and for heavy nuclei in case of protons (1.59). Secondly, the radius values for light and heavy nuclei in case of neutrons are slightly larger than the experimental value which should be in the range [1.2-1.3]. Finally, the value of $R_{C}$, the charge radius of protons is almost half of the experimental value for both the light and heavy groups. Using the final parameter values given in Table 4 after grouping all nuclei across the periodic table, neutron and proton single particle energies are computed for ${ }^{48} \mathrm{Ca}$ - a light nucleus, using both Yukawa and WoodsSaxon potential. Further, these energies are compared with experimental values and corresponding \% errors are calculated which are given in Table 5 and minimum chi- square values are shown in bold. It indicates that while Yukawa potential works well for proton states, taking light nuclei parameters with chi-square value 0.03 but WoodsSaxon shows better performance for neutron states as well as for proton states with chi-square value 0.22 and 0.01 respectively. It is also clear that Woods-Saxon potential fares better in both cases when model parameters for all nuclei are considered. Similarly, a heavy nucleus ${ }^{132} \mathrm{Sn}$ is considered and single particle energy states are calculated for both neutron and protons respectively which are compared with experimental data and is presented in Table 6. One should note that Woods-Saxon shows better performance for both heavy and light nuclei as compared to Yukawa potential shown by highlighting chi-square in bold. 
Table 5: Comparison of Yukawa and Woods-Saxon energies with the experimental values for ${ }^{48}$ Ca nucleus.

\begin{tabular}{|c|c|c|c|c|c|c|c|c|}
\hline & & $\begin{array}{l}\text { Exp. } \\
(\mathrm{MeV}) \\
{[14]}\end{array}$ & $\begin{array}{l}\text { Yukawa } \\
(\mathrm{MeV}) \\
\text { (light } \\
\text { nuclei } \\
\text { parameter) }\end{array}$ & \%error & $\begin{array}{l}\text { Yukawa }(\mathrm{MeV}) \\
\text { (all nuclei } \\
\text { parameters) }\end{array}$ & \%error & $\begin{array}{l}\text { Woods- } \\
\text { Saxon (MeV) } \\
\text { (all nuclei } \\
\text { parameters) }\end{array}$ & \%error \\
\hline \multirow{5}{*}{ Neutron } & $1 \mathrm{~d} 5 / 2$ & -15.61 & -18.53 & 18.71 & -14.02 & -10.19 & -18.07 & 13.84 \\
\hline & $2 s 1 / 2$ & -12.55 & -17.03 & 35.70 & -11.98 & -4.54 & -14.14 & 13.31 \\
\hline & $1 \mathrm{~d} 3 / 2$ & -12.53 & -16.61 & 32.56 & -10.82 & -13.65 & -13.84 & 8.62 \\
\hline & $1 \mathrm{f} 7 / 2$ & -10.00 & -11.07 & 10.70 & -5.89 & -41.10 & -8.72 & 16.40 \\
\hline & & \multicolumn{2}{|c|}{ Chi-square error } & 0.90 & & 0.53 & & 0.22 \\
\hline \multirow{3}{*}{ Proton } & $1 \mathrm{~d} 5 / 2$ & -21.47 & -20.34 & 5.26 & -13.59 & 36.70 & -20.63 & 3.91 \\
\hline & $1 \mathrm{~d} 3 / 2$ & -16.18 & -15.48 & 4.33 & -10.00 & 38.20 & -16.55 & 2.29 \\
\hline & $2 s 1 / 2$ & -16.1 & -15.81 & 1.80 & -10.57 & 34.35 & -16.12 & 0.12 \\
\hline & & \multicolumn{2}{|c|}{ Chi-square error } & 0.03 & & 2.38 & & 0.01 \\
\hline
\end{tabular}

Table 6: Comparision of Yukawa and Woods-Saxon energies with the experimental values for $\mathrm{Sn}^{-132}$ nucleus.

\begin{tabular}{|c|c|c|c|c|c|c|c|c|}
\hline & & $\begin{array}{l}\text { Exp. } \\
(\mathrm{MeV}) \\
{[14]}\end{array}$ & $\begin{array}{l}\text { Yukawa } \\
\text { (MeV) } \\
\text { (heavy } \\
\text { nuclei } \\
\text { parameters) }\end{array}$ & \%error & $\begin{array}{l}\text { Yukawa } \\
(\mathrm{MeV}) \\
\text { (all nuclei } \\
\text { parameters) }\end{array}$ & \%error & $\begin{array}{l}\text { Woods- } \\
\text { Saxon } \\
(\mathrm{MeV}) \\
{[11]}\end{array}$ & \%error \\
\hline \multirow[t]{7}{*}{ Neutron } & $1 \mathrm{~g} 7 / 2$ & -9.75 & -8.85 & 9.23 & -11.07 & 13.54 & -9.57 & 1.85 \\
\hline & $2 \mathrm{~d} 5 / 2$ & -8.97 & -12.00 & 33.78 & -13.13 & 46.38 & -9.23 & 2.90 \\
\hline & $3 s 1 / 2$ & -7.64 & -9.88 & 29.32 & -11.29 & 47.77 & -7.33 & 4.06 \\
\hline & $1 \mathrm{~h} 11 / 2$ & -7.54 & -10.14 & 34.48 & -9.85 & 30.64 & -6.76 & 10.34 \\
\hline & $2 \mathrm{~d} 3 / 2$ & -7.31 & -8.30 & 13.54 & -10.21 & 39.67 & -7.15 & 2.19 \\
\hline & & \multicolumn{2}{|c|}{ Chi-square error } & 0.56 & & 1.14 & & 0.02 \\
\hline & $2 \mathrm{p} 1 / 2$ & -16.01 & -12.07 & 24.61 & -12.78 & 20.17 & -16.01 & 0.00 \\
\hline \multicolumn{9}{|l|}{ Proton } \\
\hline & $1 \mathrm{~g} 9 / 2$ & -15.71 & -10.56 & 32.78 & -12.18 & 22.47 & -15.36 & 2.23 \\
\hline & & \multicolumn{2}{|c|}{ Chi-square error } & 1.33 & & 0.72 & & 0.004 \\
\hline
\end{tabular}




\section{Conclusions}

The nuclear potential has been modeled from first principles by using Yukawa potential and the obtained curve which is similar in appearance to that of Woods-Saxon curve has been utilized in TISE to solve for single particle energies of both neutrons and protons for various doubly magic nuclei across the periodic table. The model parameters have been optimized using variational Monte-Carlo technique by minimizing chi-square value between simulated and experimental data. These parameters have been obtained for each nucleus and also for groups of nuclei classified as light, heavy and all. It has been found that even though each of the nuclei's data could independently converge closely to experimental values with their own set of parameters, the performance is not commendable when model parameters obtained for all nuclei are considered. The Yukawa model performs better in some cases for light nuclei as compared to the heavy group, but overall it is observed that the performance of Woods-Saxon potential is superior.

\section{References}

[1] R. Velusamy, Resonance 12, 12 (2007). https://doi.org/10.1007/s12045-007-0121-3

[2] K. L. Heyde, The Nuclear Shell Model, Springer, Berlin, Heidelberg, 1994.

[3] B. Buck, A. C. Merchant and S. M. Perez, Physical Review C 45, 2247 (1992).

https://doi.org/10.1103/PhysRevC.45.2247
[4] A. Bohr and B. R. Mottelson, Nuclear Structure, World Scientific, Singapore, 1998.

[5] N. Gauthier and S. Sherrit, Am. J. Phys. 59, 1144 (1991). https://doi.org/10.1119/1.16626

[6] F. F. Marsiglio, Am. J. Phys. 77, 253 (2009). https://doi.org/10.1119/1.3042207

[7] B. A. Jugdutt and F. Marsiglio, Am. J. Phys. 81, 343 (2013). https://doi.org/10.1119/1.4793594.

[8] O. S. K. S. Sastri, et al., Phys. Educ 36, 1 (2019).

[9] A. Sharma and O. S. K. S. Sastri, Eur. J. Phys. 41, $055402(2020)$ https://doi.org/10.1088/1361-6404/ab988c.

[10] O. S. K. S. Sastri et al., Phys. Educ. (2021) (accepted for publication)

[11] A. Sharma, S. Gora, J. Bhagavathi and O. S. K. S. Sastri American Journal of Physics 88, 576 (2020). https://doi.org/10.1119/10.0001041

[12] A. Sharma and O. S. K. S. Sastri, IndiaRxiv. (Dec. 28, 2020). https://doi.org/10.35543/osf.io/5a6by

[13] A. Khachi, L. Kumar and O. S. K. S. Sastri, J. Nucl. Phy. Mat. Sci. Rad. A. (2021) Accepted.

[14] N. Schwierz, I. Wiedenhover and A. Volya, arXiv preprint (2007). https://arxiv.org/abs/0709.3525

\section{旬 CHITKARA}

Journal of Nuclear Physics, Material Sciences, Radiation and Applications

Chitkara University, Saraswati Kendra, SCO 160-161, Sector 9-C, Chandigarh, 160009, India

Volume 9, Issue 1

August 2021

ISSN 2321-8649

Copyright: [ $(2021$ O.S.K.S. Sastri et al.] This is an Open Access article published in Journal of Nuclear Physics, Material Sciences, Radiation and Applications (J. Nucl. Phy. Mat. Sci. Rad. A.) by Chitkara University Publications. It is published with a Creative Commons Attribution- CC-BY 4.0 International License. This license permits unrestricted use, distribution, and reproduction in any medium, provided the original author and source are credited. 